mechanisms as systemic adipokine levels did not reflect the intraarticular adipokine distribution.

Disclosure of interest None declared

\section{P087 RECIPROCAL CONTROL OF REGULATORY T LYMPHOCYTES AND NEUTROPHILS IN BOTH PHYSIOLOGICAL AND PATHOLOGICAL ENVIRONMENTS}

\begin{abstract}
${ }^{1} \mathrm{M}$ Batignes, ${ }^{1} \mathrm{~F}$ Santinon, ${ }^{1,2} \mathrm{M}-\mathrm{C}$ Boissier, ${ }^{1} \mathrm{~N}$ Bessis, ${ }^{1} \mathrm{P}$ Decker ${ }^{*} .{ }^{1}$ Inserm UMR 1125, University of Paris 13, Sorbonne Paris Cité; ${ }^{2}$ Rheumatology Department, Avicenne Hospital, AP-HP, Bobigny, France
\end{abstract}

\subsection{6/annrheumdis-2018-EWRR2018.103}

Introduction Regulatory $\mathrm{T}$ lymphocytes (Treg) play a key role in the control of autoimmunity. However, under inflammatory conditions like rheumatoid arthritis (RA), Treg become less suppressive and may shift toward a Th17 profile. On the contrary, polymorphonuclear neutrophils (PMN) are typical inflammatory cells activated in RA, although regulatory PMN sub-populations have been described. Treg and PMN are thus supposed to have opposite functions, but in both cases these functions can be reverted. Very few data are available on Treg-PMN communication in normal conditions, and even less during inflammation, especially in RA.

Objectives The aim was to analyse the interplay between Treg and PMN and their reciprocal modulation, both in physiological and pathological inflammatory environments.

Methods Splenic Treg and bone marrow PMN (C57BL/6 mice) were purified by magnetic sorting. Treg and PMN of healthy donors were freshly isolated by dextran sedimentation and magnetic sorting from peripheral blood. Co-cultures (1:1 ratio) were unstimulated or exposed to anti-CD3/anti-CD28 antibodies and/or LPS to stimulate Treg and/or PMN. CD4 ${ }^{+} \mathrm{FoxP}^{+}$ Treg (mouse and human), $\mathrm{Ly} 6 \mathrm{G}^{+}$(mouse) and $\mathrm{CD}^{+} 6 \mathrm{~b}^{+}$ (human) PMN were identified by flow cytometry. Cell activation was studied using antibodies against CD39, CD25, CTLA-4 (Treg) and CD11b (PMN). Treg maintenance was evaluated as the frequency of FoxP3 expression among $\mathrm{CD}^{+}$ cells, and their proliferation by CFSE staining followed by flow cytometry analysis. Co-cultures were performed using Transwell $(0.4 \mu \mathrm{m})$ to determine the respective involvement of soluble mediators/cell-contacts. Cytokine levels were quantified in culture supernatants by ELISA. Collagen-induced arthritis (CIA) was induced in $\mathrm{C} 57 \mathrm{BL} / 6$ mice by immunisation with chicken type II collagen in complete Freund's adjuvant at days 0 and 21 .

Results Without stimulation of both Treg and PMN from normal mice, no effect on any cell type was observed in co-culture. In contrast, co-culture of activated Treg with activated PMN resulted in increased maintenance of Treg with higher CTLA4 but lower CD39 expression, sustained PMN activation evidenced by CD11b up-regulation, and higher secretion of MIP-2, IL-6 and IL-17 but not IFN- $\gamma$ in normal mice. All these effects were lost in transwell experiments. Nevertheless, transfer of supernatants from LPS-activated PMN also partly increases Treg maintenance. In addition, co-cultures led to Treg proliferation. Similar results were observed in co-cultures of activated Treg/PMN isolated from CIA mice. Likewise, human Treg-PMN co-cultures led to enhanced Treg maintenance with higher expression of CTLA4/CD25 in a cell contact-dependent manner. Secretion of both IL-8 and IL-10 was enhanced in co-cultures.
Conclusions Our results show that cross-talk between Treg and PMN mainly leads to an activation of both cell types in normal or inflammatory conditions. Although cell contacts are clearly required, soluble mediators are also involved. Whether these synergistic interactions lead to a global suppressive or inflammatory milieu with functional modulation of either partner needs to be determined.

Disclosure of interest None declared

\section{P088 TGFB BOUND TO GARP PROMOTES ACETYLATION- MEDIATED FOXP3 PROTEIN STABILISATION}

P Lehmkuhl*, B Zapp, H Schulze-Koops, A Skapenko. Sektion Rheumatologie und Klinische Immunologie, Medizinische Klinik und Poliklinik IV, Universität München, Munich, Germany

\subsection{6/annrheumdis-2018-EWRR2018.104}

Introduction Regulatory $\mathrm{T}$ cells (Tregs) play a critical role in maintaining homeostasis and limiting autoimmunity. The transcription factor Forkhead box P3 (Foxp3) has been identified as the key regulator of Treg function and development. Its activity and stability are tightly regulated by acetylation dependent on the interplay between histone deacetylases (HDAC) and histone acetyltransferases (HAT). Tregs express distinctively on their cell surface glycoprotein A repetitions predominant (GARP). GARP binds latent $\mathrm{TGFb}$ and provides to Tregs an available pool of TGFb. The function of this $\mathrm{TGFb}$ source for Tregs has not been investigated yet. Tregderived $\mathrm{TGFb}$ might on the one hand be involved in mediating suppressive functions of Tregs and on the other hand be important for maintaining Treg homeostasis.

Objectives To analyse the function of surface TGFb bound to GARP utilising GARP-deficient Tregs from conditional CD4 specific GARP knockout mice.

Methods CD4 positive CD25 + or CD25-T cell from GARP-deficient or wild type mice were purified using MACS separation and stimulated with anti-CD3 and anti-CD28 in the presence or absence of $\mathrm{TGFb}$ for different times. mRNA expression profile was evaluated in non-stimulated and stimulated cells by Affymetrix gene array analysis and confirmed by real-time PCR. Acetylation, Foxp 3 protein expression and Smad2/3 phosphorylation were assessed by intracellular flow cytometry. Half-life of Foxp3 was evaluated in cell cultures using cycloheximid. Acetylation of Foxp3 was determined by fluorescence resonance energy transfer (FRET) utilising a FRET antibody pair for Foxp3 and acetylated lysine. The regulatory capacity of Treg was assessed in vivo in reconstitution experiments of Rag-deficient mice with CD25CD4 T cells or bone marrow cells.

Results GARP-deficient Tregs exhibited a markedly diminished intracellular protein acetylation. Acetylation of Foxp3 was also significantly lower. Consistently, expression of a Treg specific HDAC, HDAC9, was up regulated in Tregs lacking GARP. Both acetylation and HDAC9 expression levels could be restored by addition of exogenous $\mathrm{TGFb}$. In this regard basal phosphorylation of TGFb-dependent transcription factors Smad2/3 was diminished in Tregs lacking GARP. Further analysis revealed an unstable phenotype of GARP-deficient Tregs characterised by a shorter half-life of Foxp3, a faster loss of Foxp3 in response to anti-CD3/28 stimulation and restricted regulatory capacity.

Conclusions Lack of GARP on the cell surface results in a reduced $\mathrm{TGFb}$ availability and in a decreased $\mathrm{TGFb}$ signalling in CD4 Tregs. Diminished TGFb signalling leads in turn to an 
elevated HDAC9 expression and to a decreased Foxp3 acetylation. Lower acetylated Foxp3 determines an instable phenotype of GARP-deficient Tregs and their failure to proper regulate an immune reaction.

Acknowledgements This work supported by DFG grants SK59/ 09-1 and Schu1683/10-1, and by BMBF Projekt Arthromark 01Ec1401B.

Disclosure of interest None declared

\section{P089 CHARACTERISATION OF CHEMOKINE RECEPTORS AND MIGRATION OF REGULATORY B CELLS IN PATIENTS WITH RHEUMATOID ARTHRITIS AND IN HEALTHY DONORS}

${ }^{1,2,3} \mathrm{R}$ Audo*, ${ }^{1,2,3} \mathrm{~K}$ Schreiber, ${ }^{1,2,3}$ B Combe, ${ }^{1,2,3}$ J Morel, ${ }^{1,2,3} \mathrm{C}$ Daien. ${ }^{1} / \mathrm{GMM}$ UMR5535; ${ }^{2}$ Lapeyronie Teaching Hospital; ${ }^{3}$ Montpellier University, Montpellier, France

\subsection{6/annrheumdis-2018-EWRR2018.105}

Introduction B cells are pathogenic players in the development of rheumatoid arthritis (RA). More recently, it has been shown that $\mathrm{B}$ cells can also have regulatory functions, mainly through the secretion of interleukin-10 (IL-10). We found a decrease of IL10 +B (B10) cells in patients with early Rheumatoid Arthritis (RA) and B10 were inversely correlated with disease activity (DAS28), inflammation and rheumatoid factor, suggesting a key role at the initial phase of RA. Chemokines and chemokine receptors (CR) orchestrate migration of immune cells in physiological and pathological conditions. We hypothesise that CR could be involved in B10 functions and characterisation.

Objectives We aim to assess expression of CR on B10 and to understand their migration into the joint.

Methods Peripheral blood mononuclear cells (PBMCs) from 10 RA patients and 10 controls (HC) were activated 24 hours with $\mathrm{CpG}$ to generate B10 cells. We compared expression of several CR between B10 cells and IL- $10^{\text {neg }}$ B cells by FACS analysis. For functional test, B10 cells and IL-10 ${ }^{\text {neg }} \mathrm{B}$ were sorted by using secretion assay (Miltenyi) and the ability to migrate in response to CCL21, CCL22, CXCL11, CXCL12 or CXCL13 was evaluated by using migration assay in $5 \mu \mathrm{M}$ Transwell chambers. The presence of B10 cells in RA synovial tissue was evaluated by immunohistochemistry.

Results We found that B10 cells from HC differentially expressed several CR than IL- $10^{\text {neg }} \mathrm{B}$ cells, There were a strong decrease of CXCR5 and CXCR7 expression, and a strong increase of CCR4 expression in B10 cells $(p<0.001)$ compared to IL- $10^{\text {neg }}$ B cells. Functional impact of differential expression was tested by migration assay. Among chemokines tested,CXCL13, CXCR5-ligand, attracted more B10 cells than IL-10 ${ }^{\text {neg }} B$ cells $(n=7)$. More importantly, our results suggest that CR receptor profile on B10 cells is different between HC and RA $(n=10)$. Preliminary results showed that only a few B10 can be found in RA synovial tissue among B cells. One can hypothesised that the different expression profile of CR on B10 cells of RA patients might explain a defect in joint migration, and thus promoting uncontrolled inflammation.

Conclusions B10 cells have a specific CR profil compared to IL- $10^{\text {neg }} B$ cells. The differential expression of CR on RA B10 compared to HC might explain a defect in joint migration. Understanding of B10 migration is an important issue and could be used in the future to drive regulatory B cells into the joints as a new therapeutic approach for RA.
Disclosure of interest None declared

\section{P090 DIFFERENCE BETWEEN PALINDROMIC RHEUMATISM AND RHEUMATOID ARTHRITIS AT THE LEVEL OF GENE EXPRESSION}

S Ajaib*, A Droop, K Mankia, P Emery, F Ponchel. Leeds Institute of Rheumatic and Musculoskeletal Medicine, University of Leeds, Leeds, UK

\subsection{6/annrheumdis-2018-EWRR2018.106}

Introduction Palindromic rheumatism (PR) is characterised by recurrent, episodic attacks of articular inflammation, which resolve completely without residual joint damage. Whether PR should be considered as a prodrome of rheumatoid arthritis (RA) or as a distinct syndrome remains unclear, over 70 years since first described. ${ }^{1}$

Objectives This project investigates whether the pathogenesis of PR and RA is similar by comparing gene expression profiles from PBMCs in PR, healthy control (HC), and early RA patients.

Methods PBMCs were obtained from drug naïve PR patients during palindromic attack (flare, $\mathrm{n}=10$ ) and between attacks (non-flare, $\mathrm{n}=12$ ). 12 age/sex matched, drug nave RA patients were selected from the RA-MAP project along with $12 \mathrm{HC}$. The Illumina Human-HT-12/v4 Expression BeadChip microarray and a standard data analysis workflow, were used to establish a list of differentially expressed genes (DEGs). The STRING database was used to characterise functional associations of the top DEGs between groups. ${ }^{2}$ Only the highest confidence $(0.900)$ interactions were selected based on prior information from text-mining, experiments and databases.

Results Comparing RA and HC, STRING analysis revealed 112 interactions (from 378 DEGs); including expected associations with genes such as TNF, AP1 (JUN/FOS), chemokines/ receptors and a node including several Interferon responsive genes. Between PR non-flare and HC, 185 interactions (from 458 DEGs) were observed, featuring numerous endoplasmic reticulum and ribosomal proteins, in addition to high IL1beta/high IL-1R1, lower IL-10 and higher SOCS3. Interestingly, lower Ubiquitin $\mathrm{C}$ (UBC) was found to be a prominent feature of many of the identified interactions. Comparing inflammation between RA and PR flare, 176 interactions (from 476 DEGs) included an enhanced endoplasmic/ribosomal signature, lower ubiquitination but higher sumolation and other stress-associated proteins. SCOC3, was no longer present at the top of the DEGs list when both conditions were inflammatory. The IFN signature identified in RA was still highlighted in the associations. Finally, comparing PR non-flare and flare (10 matched pairs), 125 interactions (from 434 DEGs) were observed, primarily from pathways related again to stress, ubiquitination and DNA/RNA processing.

Conclusions Although preliminary, our findings suggest there are distinct gene expression programmes used in PR and RA, and that PR pathogenesis may involve several stress response mechanisms, which are not clearly associated with top DEGs in RA pathogenesis.

\section{REFERENCES}

1. Hench P, Rosenberg EF. Palindromic rheumatism. A 'new' oft recurring disease of joints (arthritis, periarthritis, para-arthritis) apparently producing no articular residues. Report of thirty-four cases; its relation to angio-neural arthrosis', 'allergic rheumatism' and rheumatoid arthritis. Arch Int Med 1944;73:293-321.

2. Szklarczyk D, Franceschini A, Wyder S, Forslund K, Heller D, Huerta-Cepas J, Simonovic $M$, Roth $A$, Santos $A$, Tsafou KP, Kuhn M, Bork $P$, Jensen LJ, von 